\title{
POSTER: Development of an Augmented Reality Resource System for Three-Dimensional Visualization of Constructions as of Floor Plan.
}

\author{
Mariana de Morais Ribeiro Lião \\ Student of control and automation engineering \\ Federal Institute of Education, Science and Technology (IFSP) \\ Cubatão, Brazil \\ mariana.liao@yahoo.com
}

\author{
Marcelo Pereira Bergamaschi \\ Professor at IFSP \\ Federal Institute of Education, Science and Technology (IFSP) \\ Cubatão, Brazil \\ berga@ifsp.edu.br
}

\begin{abstract}
Augmented Reality (AR) is a constantly evolving technology, and with this advancement come studies of different applications of this feature. In this project, we apply augmented reality with mobile devices in favor of the visualization processes of the construction industry. Currently, the vast majority of buildings are presented with mockups, videos or images, generating garbage in the case of mockups or being uninviting in the case of videos and images. By using Augmented Reality applications for these views, you can create a cleaner, more immersive experience. Therefore, we developed an RA beta application that consists of a virtual mockup of our college using user-friendly software. This application's development process is shown in stages throughout the article and plans are presented at the conclusion.
\end{abstract}

Keywords-mockup, architecture, 3D modeling, app.

\section{INTRODUCTION}

An Augmented Reality (AR) system can be defined in several ways [1]:

a) It is the mix of real and virtual worlds at some point of continuous reality/virtuality that connects completely real environments to completely virtual environments [2];

b) It is a system that supplements the real world with computer-generated virtual objects, appearing to coexist in the same space and has the following properties:

1. Combines real and virtual objects in the real environment;

2. Runs interactively in real-time;

3. Aligns real and virtual objects with each other;

4. It applies to all senses, including hearing,

5. Touch and strength and smell. [3].

In this project, the definition that comes closest to what is proposed would be that of Milgram [2] and partially Azuma [3].

Augmented Reality can be applied to infinite areas, such as the construction area to which this project is directed. The manufacture of the usual models used today whether physical or virtual have cons that can be avoided if you choose to use AR. Physical models have a high material cost, have a static and far-from-reality view, and are discarded after use, generating unnecessary waste. On the other hand, virtual models have low cost and can be dynamic, but still have distance from the viewer, away from the real experience. Applying the resources of the AR will make the model cheaper, can be immersive and interactive resulting in a better visualization, being a mix of the real world with the virtual one, besides not generating garbage.

By developing a system with Augmented Reality features for three-dimensional visualization of buildings from floor plans, you can deliver a more immersive and real new experience to the user. For this, 3D modeling software is used to develop virtual objects (home, building, furniture), a game engine for application development and extension (Vuforia) for linking AR resources [4] together with an SDK, these platforms have facilitated the process of producing applications with their various tools that have continuous updates for constant improvement along with their intuitive interface that speeds learning [5].

\section{OBJECTIVE}

The objective is to develop a system with Augmented Reality (AR) resources for the three-dimensional visualization of buildings from floor plans to provide construction companies and students in the area, a new form more sustainable of visualization for projects. This application would give better conditions to a user who wants to invest in a property, to visualizes how it will be built and can immerse in the property and the rooms in a virtual way. With this, the user would be able to opine or decide on certain changes in the works of his future property.

To this end, we intend to develop a beta application of the feature, which is an Augmented Reality view of our campus building.

\section{MATERIALS AND METHODS}

We will use software such as ArchiCAD for building modeling, Unity 3D which is the 2D and 3D game development platform created by Unity Technologies which is currently the most used by developers [6] to develop the application along with your Vuforia extension to include AR features and Visual Studio for screen deployment.

To put the project into practice required a computer to use all the software mentioned above and a smartphone with the Android operating system to install the application BETA.

In addition to research for theoretical background using journals, books and academic articles. 


\section{RESULTS AND DISCUSSION}

The first step was to analyze the floor plan received from the Campus engineer in AutoCAD format and use it as a drawing base to begin 3D modeling.

The second step was to adjust the floor plan to the current Campus situation by adding these details to ArchiCAD itself. In Figure I we can see the design developed above the initial floor plan. In this process the three-dimensional version is already automatically generated.

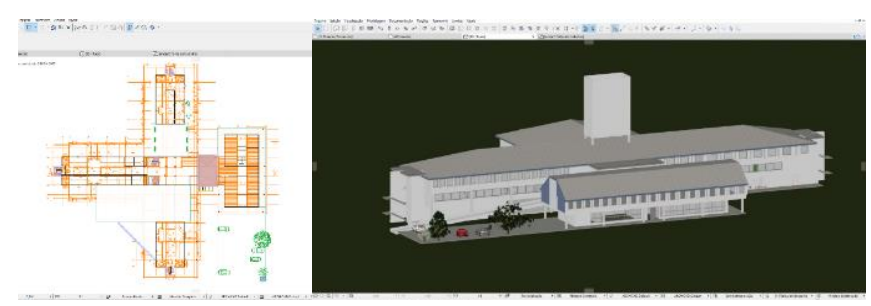

Fig. 1. Start Of 3d Modeling.

As every Augmented Reality application requires an image target whose function is to be the key that shows the respective $3 \mathrm{D}$ modeling, the ground floor plan design of the Institute was used as a marker (Figure II).

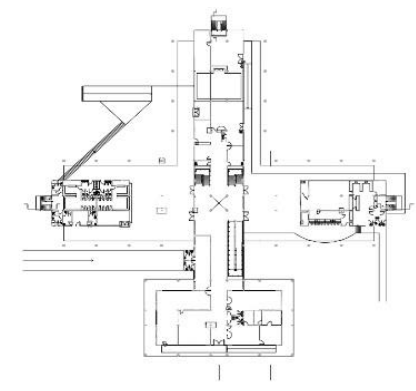

Fig. 2. Image Target.

The application was developed in Unity 3D software with the extension of Vuforia. Thus, when the camera captures this marker, the 3D modeling of the building is shown above. Finally, the mobile beta application was developed with the inclusion of scripts in $\mathrm{C}$ \# language, being shown with print screens of the application containing the menu where it can be selected to view the external structure of the building or an example of a classroom using the same marker (Figure III).

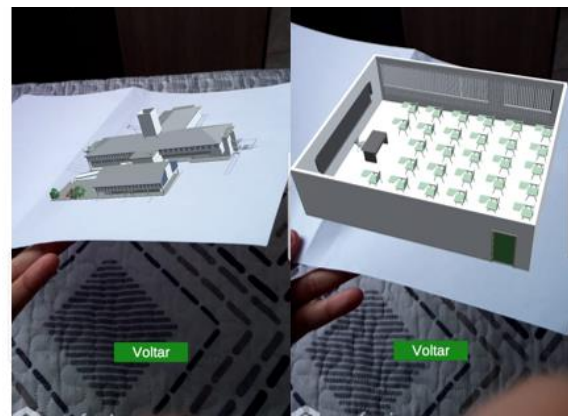

Fig. 3. Beta Application Of Augmented Reality For Mobile Devices.

It is noteworthy that the proportion of $3 \mathrm{D}$ modeling is related to the size of the marker, so if it is printed on a large scale, the virtual model will be seen proportionally.

\section{CONCLUSIONS}

By developing this project of an Augmented Reality application for visualization of 3D floor plans of civil construction, it can be shown the feasibility of its use taking into account the ease of its creation, mainly because the 3D modeling is common in the daily lives of many architects, and development has no advanced level of difficulty.

With this Beta application you can see the exterior structure of the building, the next process, which is already underway, includes application improvements and the addition of more specific views, such as our immersion option in our labs and auditoriums.

\section{ACKNOWLEDGMENT}

I thank all the professionals who helped me in some way in the development of this project, as well as the educational institution that also supported me in this path. Always thank my family who supported me during this journey.

And especially to my mentor and professor, Bergamaschi, who guided me and shared his knowledge so that together we could produce this project.

\section{REFERENCES}

[1] K. Cláudio; S. Robson. Realidade Virtual e Aumentada: Conceitos, Projeto e Aplicações. Rio de Janeiro: Pré-Simpósio IX Symposium on Virtual and Augmented Reality, 2007.

[2] M. Paul. et. al. Augmented Reality: A Class of Displays on the RealityVirtuality Continuum. Telemanipulator and Telepresence Technologies, SPIE, 1994, V.2351, p. 282-292.

[3] A. Ronald. et al. Recent Advances in Augmented Reality. IEEE Computer Graphics and Applications, v .21, n.6, p. 34-47, 2001.

[4] B. Marcelo; M. Thamires. Estudo sobre a utilização de VUFORIA e Unity 3D com RA para dispositivos móveis. Ln DCC UFMG. 2018. Accessed on: July 10, 2019 in http://www.lbd.dcc.ufmg.br/colecoes/wrva/2014/031.pdf

[5] C. Ryan. Unity 3D Game Development by Example. United Kingdom: Packt Publishing Ltd.R. Nicole, "Title of paper with only first word capitalized,” J. Name Stand. Abbrev., in press. 2010

[6] TNW DEALS. This engine is dominating the gaming industry right now . In The Next Web website. 2016. Accessed on: July 10, 2019 in https://thenextweb.com/gaming/2016/03/24/engine-dominatinggaming-industry-right-now 